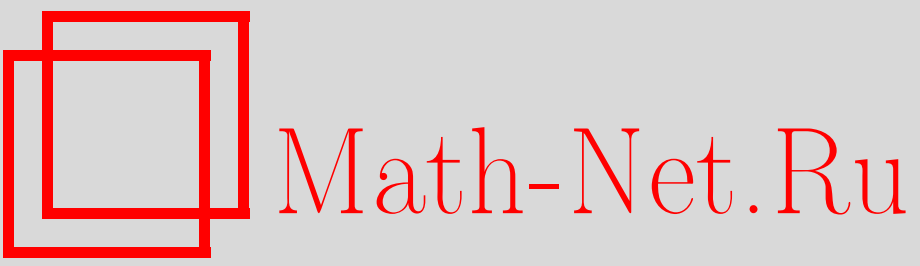

Б. И. Ботвинник, В. М. Бухштабер, С. П. Новиков, С. А. Юзвинский, Алгебраические аспекты теории умножений в комплексных кобордизмах, $У M H, 2000$, том 55, выпуск 4, 5-24

DOI: https://doi.org/10.4213/rm312

Использование Общероссийского математического портала Math-Net.Ru подразумевает, что вы прочитали и согласны с пользовательским соглашением

http://www. mathnet.ru/rus/agreement

Параметры загрузки :

IP: 54.89 .56 .158

26 апреля 2023 г., 15:44:25 


\title{
АЛГЕБРАИЧЕСКИЕ АСПЕКТЫ ТЕОРИИ УМНОЖЕНИЙ В КОМПЛЕКСНЫХ КОБОРДИЗМАХ
}

\author{
Б. И. Ботвинник, В. М. БУХштАБЕР, С. П. Новиков, С. А. ЮЗВинСкий
}

Рассмотрена общая задача классификации всех стабильных, ассоциативных умножений в теории комплексных кобордизмов. Показано, что эта задача сводится к теории алгебры Хопфа $S$ (алгебры Ландвебера-Новикова), действующей в двойственной алгебре Хопфа $S^{*}$ с выделенной “топологически целочисленной” частью $\Lambda \subset S^{*}$, которая соответствует алгебре комплексных кобордизмов точки. В терминах представлений алгебры $S$ построена формальная группа и ее логарифм. Введено понятие одномерного представления алгебры Хопфа. Приведен ряд примеров таких представлений, подсказанных известными топологическими и алгебраическими результатами. Введены и изучены операторы разностной производной в коммутативном, ассоциативном кольце без делителей нуля. Описан ряд важных примеров операторов разностной производной, естественно возникших в задачах анализа, теории представлений и некоммутативной алгебры. Особое внимание уделено операторам деления на необратимьй элемент кольца. Дано несколько конструкций новых ассоциативных умножений (в том числе и некоммутативных) при помощи операторов разностной производной. В качестве приложений описаны классы новых ассоциативных умножений в теории комплексных кобордизмов.

Библиография: 23 названия.

\section{СОДЕРЖАНИЕ}

1. Введение. Комплексные кобордизмы и алгебра ...................... 5

2. Операторы деления и умножения …................................. 14

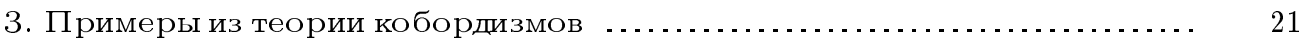

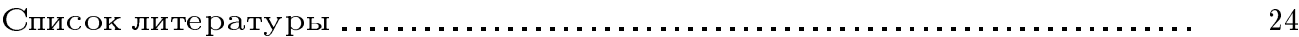

\section{1. Введение. Комплексные кобордизмы и алгебра}

Комплексные кобордизмы (бордизмы) $U^{*}(X), U^{*}(X, Y)$ представляют собой общеизвестную в топологии экстраординарную теорию (ко)гомологий, где дуальные классы бордизмов $u \in U_{k}(X)$ представлены отображениями замкнутых $U$-многообразий в пространство $X, f: M^{k} \rightarrow X$ (унитарная структура задается в стабильном расслоении, нормальном к $\left.M^{k} \subset \mathbb{R}^{n+k}\right)[1]$. Все кобордизмы $U^{*}(X, Y)$ - это коммутативные ассоциативные $\mathbb{Z}$-градуированные кольца с единицей $1 \in U^{0}(X)$. Bсе $U^{*}(X, Y)$ - это модули над алгеброй кобордизмов точки $U^{*}(p t)=\Omega_{U}^{*}=\Lambda$. Согласно теореме Милнора-Новикова (см. [2]-[4]) это кольцо изоморфно кольцу полиномов от четномерных образующих: $\Omega_{U}^{*}=\mathbb{Z}\left[u_{2}, u_{4}, u_{6}, \ldots\right]=\Lambda, \operatorname{deg} u_{2 k}=-2 k$. 
ЗАДАЧА. Дать классификацию всех стабильных ассоциативных умножений в теории комплексных кобордизмов (для конечных комплексов $X$ ).

Поясним, что мы подразумеваем под термином “умножение в теории кобордизмов": это - билинейная операция $u \circ v=\Phi(u \otimes v)$,

$$
\Phi: U^{*}(X) \otimes U^{*}(X) \rightarrow U^{*}(X)
$$

определенная для всех пространств $X$, всех элементов $u, v \in U^{*}(X)$ и коммутируюшая с непрерывными отображениями $f: X \rightarrow Y$,

$$
f^{*}(u) \circ f^{*}(v)=\Phi\left(f^{*}(u) \otimes f^{*}(v)\right)=f^{*} \Phi(u \otimes v)=f^{*}(u \circ v) .
$$

Следует пояснить также термин "стабильное умножение" - оно должно коммутировать с изоморфизмом надстройки $s$. Имеется отмеченный элемент $\mu \in U^{1}\left(S^{1}\right)$ такой, что $s: U^{*}(X) \rightarrow \widetilde{U}^{*}(\Sigma X), u \rightarrow \mu u=s(u)$, есть изоморфизм надстройки, где $S^{1}$ - окружность, $\Sigma X=S^{1} \wedge X$ - надстройка над пространством $X$ и $\widetilde{U}^{*}(\Sigma X)=$ $U^{*}(\Sigma X, p t)$. Здесь и далее $\wedge$ - символ приведенного ("тензорного") произведения в категории пространств с отмеченньми точками, $X \wedge Y=X \times Y / X \vee Y$. Мы требуем стабильности нового умножения $\Phi(u \otimes v)=u \circ v$, исходя из условия:

$$
\mu(u \circ v)=(\mu u) \circ v=(-1)^{q_{1}} u \circ(\mu v),
$$

где $q_{1}=\operatorname{deg} u$, а знаком $u v$ обозначается обычное умножение. Очевидно, оно стабильно.

Напомним, что теория комплексных кобордизмов является модулем над "алгеброй Стинрода" всех операций $A^{U}$, т.е. линейных операторов $a: U^{*}(X) \rightarrow U^{*}(X)$, коммутируюших с непрерывньми отображениями и надстройкой (стабильных). Эта алгебра, вычисленная в [5], состоит из двух частей:

1. Кольцо $\Lambda=\Omega^{U} \subset A^{U}$, состоящее из операторов умножения на "скаляр" $u \rightarrow \lambda u$, $\lambda \in \Lambda$.

2. Алгебра Ландвебера-Новикова $S$ [5], [6]. Это - алгебра Хопфа над $\mathbb{Z}$, которая $\mathbb{Z}^{+}$-градуирована, $S=\sum_{j \geqslant 0} S^{j}$, с базисом $s_{w} \in S$, где $S^{0}=\mathbb{Z}$ и $w=\left(k_{1}, \ldots, k_{l}\right)$ это либо неупорядоченный набор положительных целых чисел $k_{j} \in \mathbb{Z}^{+}$, либо символ пустого множества, $s_{\varnothing}=s_{0} \in S^{0}, s_{0}=1$ и $\operatorname{deg} w=2 \sum k_{q}$.

Коумножение (диагональ) имеет вид

$$
\Delta s_{w}=\sum_{\left(w^{\prime}, w^{\prime \prime}\right)=w} s_{w^{\prime}} \otimes s_{w^{\prime \prime}}
$$

При этом алгебра Хопфа $S$ действует на всех $U^{*}(X)$ (в том числе на $\Lambda=$ $\left.U^{*}(p t)=\Omega^{U}\right)$, преврашая их в "модули Милнора",

$$
s_{w}(u v)=\sum_{\left(w^{\prime}, w^{\prime \prime}\right)=w} s_{w^{\prime}}(u) s_{w^{\prime \prime}}(v) .
$$

Примитивные элементы $s_{(n)}$ порождают алгебру Ли: $\left[s_{(n)}, s_{(m)}\right]=(m-n) s_{(m+n)}$. 
Так называемые геометрические кобордизмы $x \in U^{2}(X)$ определяются исходя из требования

$$
s_{(k)}(x)=x^{k+1}, \quad k \geqslant 0 \text { и } s_{w}(x)=0, \quad w=\left(k_{1}, \ldots, k_{l}\right), \quad l>1 .
$$

Представление алгебры $S$ (и всей алгебры $A^{U}$ ) является асимптотически точнылм на произведении большого числа геометрических кобордизмов:

$$
s \rightarrow s\left(x_{1} x_{2} \cdots x_{N}\right), \quad s \in S
$$

т.е. для любого элемента $s \in S$ размерности $2 m$ найдется такое число $N>m$, что $s\left(x_{1} x_{2} \cdots x_{N}\right)=0$ эквивалентно $s=0$. Действие алгебры $S$ на кольце $\Lambda$ является точным представлением. Оно впервые вычислено в [5]. В работе [7] найдена интерпретация этого действия через дифференциальные операторы на бесконечномерной группе Ли формальных диффеоморфизмов прямой. В работах [8], [9] развивалась теория операторов на квантовых группах, основанная на обобщении этого примера. Здесь $A^{U}=(\Lambda S)^{\wedge}$, т.е. пополнение рядами вида

$$
\sum_{j=0}^{\infty} \lambda_{j} s_{w_{j}} \in A^{U}, \quad \lambda_{j} \in \Lambda, \quad s_{w_{j}} \in S
$$

где $\operatorname{deg} w_{j} \rightarrow+\infty$, когда $j \rightarrow \infty$. При этом правило коммутирования определяется представлением алгебры $S$ на алгебре $\Lambda$. Имеет место вложение $\Lambda \subset S^{*}: \lambda\left(s_{w}\right)=$ $\varepsilon s_{w}(\lambda)$, где $\lambda \in \Lambda$ и $\varepsilon: \Lambda \rightarrow \mathbb{Z}$ - аугментация, $\varepsilon(1)=1$ и $\varepsilon(\lambda)=0$, если $\operatorname{deg} \lambda<0$. Здесь $S^{*}$ - это алгебра Хопфа, двойственная к $S ; \Lambda \otimes Q=S^{*} \otimes Q$. Очевидно, кольцо $S^{*}$ - это кольцо полиномов над $\mathbb{Z}$ от образующих $s_{k}^{*}$ таких, что $\left(s_{k}^{*}, s_{w}\right)$ равно единице, если $w=(k)$, и равно нулю в противном случае. Алгебраическое описание подкольца $\Lambda \subset S^{*}$ мы дадим ниже.

Лемма 1. Всякое стабильное умножсение в теории комплексных кобордизмов задается формальным рядом вида

$$
\widehat{\Phi}=\sum \lambda_{i j} s_{w_{i}} \otimes s_{w_{j}}
$$

где $\lambda_{i j} \in \Lambda, \operatorname{deg} w_{i} \rightarrow \infty, \operatorname{deg} w_{j} \rightarrow \infty, i, j \rightarrow \infty$, так что умножсение определяется формулой

$$
u \circ v=\Phi(u \otimes v)=\sum \lambda_{i j} s_{w_{i}}(u) s_{w_{j}}(v)
$$

$u, v \in U^{*}(X)$.

Все коэффиииенты ряда $\widehat{\Phi}$ однозначно определяются умножением $и$ о $v$ дя $u, v \in \Lambda=U^{*}(p t)=\Omega^{U}$. 
ДокаЗАтельство. Пусть $x(N) \in U^{2 N}\left(M U_{N}\right)$ - канонический класс Тома пространства Тома $M U_{N}$ универсального комплексного $N$-мерного расслоения $\eta_{N} \rightarrow$ $B U(N)$.

Известно, что $U^{*}\left(M U_{N}, p t\right)$ является одномерным $A^{U}$-модулем с образуюшим $x(N)$, причем элементы $s_{w} x(N)$, где $w=\left(k_{1}, \ldots, k_{l}\right), l \leqslant N$, вместе с $x(N)=s_{0} x(N)$ составляют его базис над $\Lambda$.

Пусть задано некоторое стабильное умножение о в теории комплексных кобордизмов. Тогда для любых $N_{1}$ и $N_{2}$ определено произведение $x\left(N_{1}\right) \circ x\left(N_{2}\right) \in U^{*}\left(M U_{N_{1}} \times\right.$ $\left.M U_{N_{2}}\right)$. В виду билинейности умножения о мы имеем:

$$
x\left(N_{1}\right) \circ x\left(N_{2}\right) \in \widetilde{U}^{*}\left(M U_{N_{1}} \wedge M U_{N_{2}}\right),
$$

и, следовательно, это произведение можно однозначно записать в виде формального ряда

$$
x\left(N_{1}\right) \circ x\left(N_{2}\right)=\sum \lambda_{i j} s_{w_{i}}\left(x\left(N_{1}\right)\right) s_{w_{j}}\left(x\left(N_{2}\right)\right),
$$

где $w_{i}=\left(k_{1}^{\prime}, \ldots, k_{l_{1}}^{\prime}\right), l_{1} \leqslant N_{1}$ и $w_{j}=\left(k_{1}^{\prime \prime}, \ldots, k_{l_{2}}^{\prime \prime}\right), l_{2} \leqslant N_{2}$. Коэффициенты $\lambda_{i j} \in \Lambda$ не зависят от $N_{1}$ и $N_{2}$, так как умножение о коммутирует с непрерьвными отображениями и стабильно. Напомним, что пространства Тома $M U_{N}$ для разных $N$ связаны отображениями $e_{N_{1}, N_{2}}: \Sigma^{2\left(N_{2}-N_{1}\right)} M U_{N_{1}} \rightarrow M U_{N_{2}}$ такими, что $e_{N_{1}, N_{2}}^{*} x\left(N_{2}\right)=$ $s^{2\left(N_{2}-N_{1}\right)} x\left(N_{1}\right)$.

Для любых элементов $u \in U^{q_{1}}(X, Y)$ и $v \in U^{q_{2}}(X, Y)$ существуют отображения

$$
f_{1}: \Sigma^{2 N_{1}-q_{1}} X / Y \rightarrow M U_{N_{1}} \text { и } f_{2}: \Sigma^{2 N_{2}-q_{2}} X / Y \rightarrow M U_{N_{2}}
$$

такие, что $f_{1}^{*} x\left(N_{1}\right)=s^{2 N_{1}-q_{1}} u$ и $f_{2}^{*} x\left(N_{2}\right)=s^{2 N_{2}-q_{2}} v$ для некоторых $N_{1}$ и $N_{2}$. Возьмем отображение

$$
f=f_{1} \wedge f_{2}: \Sigma^{2\left(N_{1}+N_{2}\right)-\left(q_{1}+q_{2}\right)} X \stackrel{f_{1} \wedge f_{2}}{\longrightarrow} M U_{N_{1}} \wedge M U_{N_{2}} .
$$

Тогда

$$
\begin{aligned}
f^{*}\left(x\left(N_{1}\right) \circ x\left(N_{2}\right)\right) & =\sum \lambda_{i j} s_{w_{i}}\left(s^{2 N_{1}-q_{1}} u\right) s_{w_{j}}\left(s^{2 N_{2}-q_{2}} v\right) \\
& =(-1)^{q_{1} q_{2}} s^{2\left(N_{1}+N_{2}\right)-\left(q_{1}+q_{2}\right)}\left(\sum \lambda_{i j} s_{w_{i}}(u) s_{w_{j}}(v)\right) .
\end{aligned}
$$

С другой стороны,

$$
\begin{aligned}
f^{*}\left(x\left(N_{1}\right) \circ x\left(N_{2}\right)\right) & =\left(f_{1}^{*} x\left(N_{1}\right)\right) \circ\left(f_{2}^{*} x\left(N_{2}\right)\right)=\left(s^{2 N_{1}-q_{1}} u\right) \circ\left(s^{2 N_{2}-q_{2}} v\right) \\
& =(-1)^{q_{1} q_{2}} s^{2\left(N_{1}+N_{2}\right)-\left(q_{1}+q_{2}\right)} u \circ v .
\end{aligned}
$$

Таким образом, первая часть леммы доказана.

Пусть теперь задано умножение $u \circ v$ на кольце $\Lambda=U^{*}(p t)$. Заметим, что если $u \in \Lambda^{-2 n}$ и $v \in \Lambda^{-2 m}$, то

$$
u \circ v=\Phi(u \otimes v)=\sum_{\substack{\operatorname{deg} w_{i} \leqslant 2 n, \operatorname{deg} w_{j} \leqslant 2 m}} \lambda_{i j} s_{w_{i}}(u) s_{w_{j}}(v) .
$$

При $\operatorname{deg} w=2 n$ мы имеем:

$$
s_{w}(u)=\varepsilon s_{w}(u)=\left\langle u, s_{w}\right\rangle,
$$

где $\varepsilon: \Lambda \rightarrow Z$ - аугментация. 
Используя описанное вьше вложение $\Lambda \subset S^{*}$ и то, что $\Lambda \otimes Q=S^{*} \otimes Q$, мы при помоши формулы (7) индукцией по $\operatorname{deg} w$ можем восстановить все коэффициенты $\lambda_{i j}$, зная умножения $u \circ v$ в кольце $\Lambda$, а также действие алгебры $S$ на нем. Лемма 1 полностью доказана.

Мы принимаем формальные ряды вида (5) за определение нового стабильного умножения в теории $U^{*}(X)$. Свойства этих умножений мы и изучаем далее.

В рамках общей теории алгебр Хопфа ситуация такова.

Задана алгебра Хопфа $S$ и модуль Милнора $\Lambda$ над ней (т.е. $S$ действует на алгебре $\Lambda$ и $s(u v)=\sum s_{i}^{\prime}(u) s_{i}^{\prime \prime}(v)$, где $\left.\Delta(s)=\sum s_{i}^{\prime} \otimes s_{i}^{\prime \prime}\right)$. Новое умножение в алгебре $\Lambda$ задается формулой (6). Задачей является классификация ассоциативных умножений такого вида.

В особо интересном случае, подсказанном теорией кобордизмов, модуль Милнора $S^{*}$ над $S$ является алгеброй Хопфа, двойственной к $S$ в базисе $s_{w}$, где $\Lambda \subset S^{*}$. При этом действие алгебры $S$ на $S^{*}$ определяется как $s(u)=R_{s}^{*}(u)$, где $R_{s}$ - это оператор правого умножения [7], [8]:

$$
R_{s}\left(s^{\prime}\right)=s^{\prime} s, \quad R_{s}: S \rightarrow S, \quad R_{s}^{*}: S^{*} \rightarrow S^{*}, \quad\left(R_{s}^{*}(u), s^{\prime}\right)=\left(u, s^{\prime} s\right) .
$$

Тем самым, задача классификации умножений в теории кобордизмов $U^{*}(X)$ сводится к теории одной алгебры $S$, действуюшей как $R^{*}$ в двойственной алгебре Хопфа $S^{*}$ с выделенной "топологически целочисленной” частью $\Lambda \subset S^{*}$.

Как описать кольцо $\Lambda \subset S^{*}$ чисто алгебраически? Для этого мы воспользуемся геометрическими кобордизмами $x \in U^{2}(X)$, определенными вьше. Рассмотрим модуль Милнора, представляющий собой кольцо $S^{*}\left[\left[x_{1}, x_{2}\right]\right]$ формальных рядов от $\left(x_{1}, x_{2}\right)$ с коэффициентами в кольце $S^{*}$. При этом мы определяем действия алгебры Хопфа $S$ на этом модуле алгебраически, требуя, чтобы $x_{1}, x_{2}$ были геометрическими элементами, т.е.:

$$
\begin{gathered}
s_{(k)}\left(x_{j}\right)=x_{j}^{k+1}, \quad s_{w}\left(x_{j}\right)=0, \quad w=\left(k_{1}, \ldots, k_{l}\right), \quad l>1, \\
s(\lambda)=R_{s}^{*}(\lambda), \quad\left(R_{s}^{*}(\lambda), s^{\prime}\right)=\left(\lambda, s^{\prime} s\right), \\
s(u v)=\sum s_{i}^{\prime}(u) s_{i}^{\prime \prime}(v), \quad \Delta=\sum s_{i}^{\prime} \otimes s_{i}^{\prime \prime} .
\end{gathered}
$$

Тем самым, действие колец $S$ и $S^{*}$ определено.

Лемма 2. Существует единственный ряд вида

$$
x=f\left(x_{1}, x_{2}\right)=x_{1}+x_{2}+\sum_{i, j \geqslant 1} \alpha_{i j} x_{1}^{i} x_{2}^{j}, \quad \alpha_{i j} \in S^{*},
$$

такой, что для геометрических әлементов $x_{1}$ и $x_{2}$ әлемент $x=f\left(x_{1}, x_{2}\right)$ является также геометрическим, т.е. действие алгебры $S$ на әлемент $x$ определяется формулой (4). 
ДокаЗАТЕльСтво. Введем оператор $S_{t}=\sum_{w \geqslant 0} s_{w} t^{w}, w=\left(k_{1}, \ldots, k_{l}\right), t^{w}=$ $t_{1}^{k_{1}} \cdots t_{l}^{k_{l}}$,где $t_{i}, i=1,2, \ldots,-$ алгебраически независимые переменные. Легко видеть, что

$$
\Delta S_{t}=\sum_{w \geqslant 0}\left(\Delta s_{w}\right) t^{w}=S_{t} \otimes S_{t} \text { или } S_{t}(u v)=S_{t}(u) S_{t}(v) .
$$

Подсчитаем значение $S_{t}(x)$ для любого геометрического элемента. Из определения геометрического элемента получаем, что $S_{t}(x)$ представляет собой ряд $\sum_{k=0}^{\infty} x^{k+1} t_{k}$, где $t_{0}=1$, который далее для краткости мы будем обозначать как $\phi_{t}(x)$. Применим оператор $S_{t}$ к обеим частям $(9)$. Мы получим

$$
S_{t}(x)=S_{t}\left(x_{1}\right)+S_{t}\left(x_{2}\right)+\sum_{i, j \geqslant 1} S_{t}\left(\alpha_{i j}\right) S_{t}\left(x_{1}\right)^{i} S_{t}\left(x_{2}\right)^{j},
$$

где $x, x_{1}, x_{2}$ - геометрические элементы, т.е. $S_{t}(x)=\phi_{t}(x), S_{t}\left(x_{1}\right)=\phi_{t}\left(x_{1}\right), S_{t}\left(x_{2}\right)=$ $\phi_{t}\left(x_{2}\right)$. Применим к формуле (10) аугментацию $\varepsilon$,

$$
\varepsilon: S^{*} \rightarrow \mathbb{Z}
$$

где $\varepsilon\left(s_{n}^{*}\right)=0$ для $n \neq 0, S^{*}=\sum_{n \leqslant 0} S_{n}^{*}$ и $\varepsilon\left(x_{1}\right)=x_{1}, \varepsilon\left(x_{2}\right)=x_{2}$.

Мы имеем:

$$
\begin{gathered}
\varepsilon S_{t}(x)=\sum \varepsilon(x)^{k+1} t_{k}, \quad \varepsilon(x)=x_{1}+x_{2}, \\
\varepsilon S_{t}\left(x_{1}\right)=\sum \varepsilon\left(x_{1}\right)^{k+1} t_{k}=\sum x_{1}^{k+1} t_{k} \\
\varepsilon S_{t}\left(x_{2}\right)=\sum \varepsilon\left(x_{2}\right)^{k+1} t_{k}=\sum x_{2}^{k+1} t_{k} .
\end{gathered}
$$

По определению, верна формула:

$$
\varepsilon S_{t}\left(\alpha_{i j}\right)=\sum_{w} \varepsilon s_{w}\left(\alpha_{i j}\right) t^{w}=\sum_{w}\left(s_{w}, \alpha_{i j}\right) t^{w} .
$$

Отсюда имеем

$$
\varepsilon S_{t}(x)=\phi_{t}\left(x_{1}+x_{2}\right)=\phi_{t}\left(x_{1}\right)+\phi_{t}\left(x_{2}\right)+\sum_{i, j} \sum_{w}\left(s_{w}, \alpha_{i j}\right) t^{w} \phi_{t}\left(x_{1}\right)^{i} \phi_{t}\left(x_{2}\right)^{j} .
$$

Обозначим $\phi_{t}\left(x_{j}\right)$ через $y_{j}$. Мы получаем $x_{j}=\phi_{t}^{-1}\left(y_{j}\right)$ и

$$
\varepsilon S_{t}(x)=\phi_{t}\left(\phi_{t}^{-1}\left(y_{1}\right)+\phi_{t}^{-1}\left(y_{2}\right)\right)=\sum_{i, j} \sum_{w}\left(s_{w}, \alpha_{i j}\right) t^{w} y_{1}^{i} y_{2}^{j} .
$$

Сравнивая коэффициенты при $y_{1}^{i} y_{2}^{j}$ в левой и правой частях, мы видим, что все значения $\left(s_{w}, \alpha_{i j}\right)$ вычислены в левой части и полностью известны. Тем самым, все $\alpha_{i j}$ однозначно определены как элементы кольца $S^{*}$, т.е. как линейные формы на $S$. Единственность доказана.

Сушествование ряда (9) следует из теории комплексных кобордизмов [5]. Чисто алгебраическое доказательство этого факта является следствием приводимой ниже леммы 3.

Лемма доказана.

Непосредственно из леммы 2 получаем 
СлЕДСТВИЕ 1. Ряд $x=f\left(x_{1}, x_{2}\right)$ (см. (9)) задает формальную группу на множестве геометрических әлементов.

ОПРЕДЕЛЕНИЕ 1 . Подкольцо $\Lambda \subset S^{*}$, порожденное над $\mathbb{Z}$ коэффициентами $\alpha_{i j} \in S^{*}$ ряда (9), назьвается кольиом комплексных кобордизмов. (Из топологии известно, что оно изоморфно кольцу полиномов, см. выше.)

ЗАмЕчАниЕ. Формальная группа в теории комплексных кобордизмов была введена в [5] геометрически. Она совпадает с формальной группой, задаваемой рядом (9), ввиду его единственности. Мы обрашаем внимание на то, что в данной работе формальная группа определена чисто алгебраически, как и кольцо $\Lambda$, исходя только из алгебры $S$.

Над кольцом $\Lambda \otimes \mathbb{Q}$ формальную группу $f\left(x_{1}, x_{2}\right)$ можно представить в виде:

$$
f\left(x_{1}, x_{2}\right)=g^{-1}\left(g\left(x_{1}\right)+g\left(x_{2}\right)\right),
$$

где $g^{-1}(g(x))=x$ и $g(x)=x+\sum b_{i} x^{i+1}, b_{i} \in \Lambda \otimes \mathbb{Q}$. Ряд $g(x)=g_{f}(x)$ назьвается логарифмом формальной группь $f\left(x_{1}, x_{2}\right)$. Использованньй выше подход позволяет ввести этот ряд также только в терминах алгебры $S$.

Лемма 3. Существует единственный ряд вида

$$
g(x)=x+\sum_{i=1}^{\infty} b_{i} x^{i+1}, \quad b_{i} \in S^{*}
$$

такой, что для любого геометрического әлемента $x$ мы имеем $s_{w} g(x)=0$ для всех $w, \operatorname{deg} w>0$. Этот ряд задает логарифм формальной группь и определяется из условия

$$
x=g(x)+\sum_{k=1}^{\infty} s_{k}^{*} g(x)^{k+1},
$$

m.е. $g^{-1}(t)=t+\sum_{k=1}^{\infty} s_{k}^{*} t^{k+1}$, где $s_{k}^{*}-$ мультипликативные образующие коль-

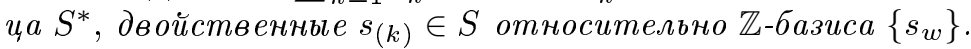

ДокАЗАТЕльство. Пусть $x$ - геометрический элемент и $g(x)=x+\sum b_{i} x^{i+1}$, $b_{i} \in S^{*},-$ такой ряд, что $s_{w} g(x)=0$ для всех $w$, где $w \neq \varnothing$. Тогда $S_{t} g(x)=$ $g(x)$, где $S_{t}=\sum s_{w} t^{w}$. Имеем: $x=g^{-1}(g(x))$. И следовательно, $\phi_{t}(x)=S_{t} x=$ $S_{t}\left[g^{-1}\right]\left(S_{t} g(x)\right)=S_{t}\left[g^{-1}\right](g(x))$, где если $g^{-1}(t)=t+\sum a_{i} t^{i+1}, a_{i} \in S^{*}$, то $S_{t}\left[g^{-1}\right]=$ $t+\sum S_{t}\left(a_{i}\right) t^{i+1}$. Пусть теперь $x_{1}$ и $x_{2}$ - геометрические элементы. Образуем ряд

$$
F\left(x_{1}, x_{2}\right)=g^{-1}\left(g\left(x_{1}\right)+g\left(x_{2}\right)\right)=x_{1}+x_{2}+\sum \alpha_{i j}^{\prime} x_{1}^{i} x_{2}^{j} .
$$

Имеем:

$$
g\left(x_{1}\right)+g\left(x_{2}\right)=g\left(F\left(x_{1}, x_{2}\right)\right)
$$

Тогда

$$
\begin{aligned}
S_{t} F\left(x_{1}, x_{2}\right) & =S_{t}\left[g^{-1}\right]\left(S_{t} g\left(x_{1}\right)+S_{t} g\left(x_{2}\right)\right) \\
& =S_{t}\left[g^{-1}\right]\left(g\left(x_{1}\right)+g\left(x_{2}\right)\right)=S_{t}\left[g^{-1}\right]\left(g\left(F\left(x_{1}, x_{2}\right)\right)\right)
\end{aligned}
$$


Используя теперь, что $S_{t}\left[g^{-1}\right](g(x))=\phi_{t}(x)$, получаем

$$
S_{t} F\left(x_{1}, x_{2}\right)=\phi_{t}\left(F\left(x_{1}, x_{2}\right)\right)
$$

Следовательно, ряд $F\left(x_{1}, x_{2}\right)$ является геометрическим элементом и поэтому, согласно лемме 2 , совпадает с рядом (9). Таким образом, если ряд $g(x)$, удовлетворяющий условиям леммы, существует, то он единственен и задает логарифм формальной группा $(9)$.

Дадим чисто алгебраическое доказательство существования такого ряда. Рассмотрим модуль Милнора, определяемьй как кольцо $S^{*}[[t]]$, со следующим действием алгебры $S$ :

$$
s_{0} t=t, \quad s_{w} t=0, \quad \operatorname{deg} w>0 \quad \text { и } s_{w}(\lambda)=R_{s}^{*}(\lambda), \quad \lambda \in S^{*} .
$$

Покажем, что ряд

$$
x=\gamma(t)=t+\sum s_{(n)}^{*} t^{n+1} \in S^{*}[[t]]
$$

является геометрическим элементом. Для этого достаточно доказать, что

$$
s_{w}\left(s_{(n)}^{*}\right)= \begin{cases}0, & \text { если } w \neq(k), \\ \gamma_{n, k}, & \text { если } w=(k),\end{cases}
$$

где $\gamma_{n, k}-$ коэффициент при $t^{n+1}$ у ряда $\gamma(t)^{k+1}$. Требуемьй результат можно теперь получить непосредственно, если воспользоваться формулой

$$
\left\langle s_{w_{2}}\left(s_{n}^{*}\right), s_{w_{1}}\right\rangle=\left\langle s_{n}^{*}, s_{w_{1}} s_{w_{2}}\right\rangle
$$

и тем фактом, что представление алгебры $S$ на произведении геометрических элементов является асимптотически точным.

Возьмем теперь $g(x) \in S^{*}[[t]]$ такой, что $g(x)=g(\gamma(t))=t$. Имеем: $x$ - геометрический элемент, и по построению $s_{w}(t)=0$, когда $\operatorname{deg} w>0$. Следовательно, ряд $g(x)$ удовлетворяет условиям леммы 3. Лемма доказана.

Результат леммы 3 методами теории комплексных кобордизмов впервые был получен в $[10]$.

ОПРЕДЕЛЕниЕ 2. Представление алгебры Хопфа $S$ в модуле Милнора $P$ над ней мы назовем одномерным, если $P$ есть алгебра полиномов (или формальных рядов) от одной образуюшей $u \in P$ над некоторым кольцом коэффициентов. Допускается нетривиальное действие алгебры $S$ на этом кольце коэффишиентов, которое в наших примерах есть $\mathbb{Z}, \mathbb{Q}, \Lambda$ или некоторьй другой подмодуль Милнора в $S^{*} \otimes \mathbb{Q}$.

Высотой представления мы назовем такое число $k$, что $u^{k+1}=0$.

Например, $P$ может быть алгеброй полиномов от одной образующей $u \in P$ с коэффициентами в кольце $\Lambda \subset S^{*}$, где $S$ действует, как указано выше.

ПримеРы. Для высоты $k=1$ мы полагаем $P=U^{*}\left(S^{2 q}\right)$ для любого $q$. Уже для $k=2$ можно построить градуированно корректные одномерные представления над $\mathbb{Z}$ лишш для случаев $\operatorname{dim} u=2,4,8$. Для $k \geqslant 3$ нам известны из топологии лишш случаи $\operatorname{dim} u=2$ или 4 , где $P=U^{*}\left(\mathbb{C} P^{k}\right)$ или $P=U^{*}\left(H P^{k}\right)$ соответственно. Случай $k=\infty$ особенно интересен. Над полем рациональных чисел мы имеем одномерные 
представления алгебры $S$ на $P=U^{*}\left(\Omega S^{2 q+1}\right) \otimes \mathbb{Q}=\Omega_{U} \otimes \mathbb{Q}[[u]]$, где $\operatorname{dim} u_{q}=2 q$ для вcex $q$.

Рассмотрим более детально случай $q=1$. Отображение $\phi: \Omega S^{3} \rightarrow \mathbb{C} P^{\infty}$, соответствуюшее образуюшему двумерной группы когомологий $H^{2}\left(\Omega S^{3}, \mathbb{Z}\right)=\mathbb{Z}$, задает геометрический элемент $u_{1}=\phi^{*}(u) \in U^{2}\left(\Omega S^{3}\right)$, где $u \in U^{2}\left(\mathbb{C} P^{\infty}\right)$ - канонический геометрический элемент. Имеем $U^{*}\left(\Omega S^{3}\right) \otimes \mathbb{Q}=\Omega_{U} \otimes \mathbb{Q}\left[\left[u_{1}\right]\right]$. Из гомотопической топологии хорошо известно отображение $\psi: \Sigma \Omega S^{3} \rightarrow S^{3}$, задаюшее элемент $\beta \in U^{2}\left(\Omega S^{3}\right)$ такой, что $s \beta=\psi^{*} \alpha$, где $\alpha$ - образующий группы $U^{3}\left(S^{3}\right)=\mathbb{Z}$, и $s-$ изоморфизм надстройки. Все операции $s_{w}$ стабильны, поэтому из того, что $s_{w} \alpha=0$, $\operatorname{deg} w>0$, вытекает, что $s_{w} \beta=0, \operatorname{deg} w>0$. В кольце $U^{*}\left(\Omega S^{3}\right) \otimes \mathbb{Q}$ элемент $\beta$ описывается рядом от геометрического элемента $u_{1}$, т.е. $\beta=g\left(u_{1}\right)$, причем $g\left(u_{1}\right)=u_{1}+\cdots$ Таким образом, в качестве следствия леммы 3 мы получаем, что $\beta$ задается рядом $g\left(u_{1}\right)$, представляюшим собой логарифм формальной групшы геометрических элементов, и $g^{-1}(\beta)=\beta+\sum_{k \geqslant 1} s_{k}^{*} \beta^{k+1}=u_{1} \in U^{2}\left(\Omega S^{3}\right)$. Обратим внимание, что $s_{k}^{*} \in S^{*}$ не принадлежат кольцу $\Lambda \subset S^{*}$ и только $(k+1) ! s_{k}^{*} \in \Lambda$ (см. [10]).

При $q>1$ описание одномерных представлений алгебры $S$ на $P=U^{*}\left(\Omega S^{2 q+1}\right) \otimes \mathbb{Q}$ получается, если использовать канонические отображения

$$
\phi_{q}: \Omega S^{2 q+1} \rightarrow \Omega S U(2 q+1) \rightarrow B U,
$$

соответствующие образующему гомотопической групшы $\pi_{2 q+1}(S U(2 q+1))=\mathbb{Z}$, где $S U(2 q+1)$ - специальная унитарная группа, и $B U$ - бесконечномерное многообразие Грассмана. При построении отображений $\phi_{q}$ используется тот факт, что согласно периодичности Ботта $B U \approx \Omega S U(\infty)$.

Важньй пример одномерного $\mathbb{Z}$-представления алгебры Хопфа $S$ (градуированного) порождается геометрическими элементами $u=x$ (см. выше) размерности $\operatorname{dim} u=2$. Пример одномерного $\Lambda$-представления алгебры Хопфа $S$ (градуированного) порождается элементами вида $u=x \bar{x}$, где $\bar{x}$ - обратньй элемент в формальной группе, $\operatorname{dim} u=4$. Здесь $s_{w}(u)$ принадлежит кольцу $\Lambda[[u]]$. На подобные элементы натягивается двузначная формальная группа, впервые замеченная в [11]. Их алгебраическая теория была построена в [12]. Для случая $\operatorname{dim} u=8, k=2$ мы можем взять $\Lambda$-представление на $P=U^{*}\left(\mathrm{CaP}^{2}\right)$, где $\mathrm{CaP} P^{2}$ - проективная плоскость Кэли. Для $k \geqslant 3$, вероятно, одномерных представлений высоты $k$ с $\operatorname{dim} u>4$ на кольцах комплексных кобордизмов пространств уже нет. Было бы полезно это доказать.

ЗАДАЧА. Дать классификацию одномерных $\mathbb{Z}$-представлений и $\Lambda$-представлений алгебры $S$, градуированно корректных, относительно замен

$$
u=\Psi(v)=v+\sum_{i \geqslant 1} \lambda_{i} v^{i+1}
$$

где $\lambda_{i} \in \mathbb{Z}$ (или $\left.\mathbb{Q}\right)$, либо $\lambda_{i} \in \Lambda$ (или $\lambda_{i} \in \Lambda \otimes \mathbb{Q}=S^{*} \otimes \mathbb{Q}$ ).

Примеры таких представлений над некоторыми подкольцами в $\Lambda \otimes \mathbb{Q}$ извлекаются из [13]. Они порождаются элементами $u_{n}=x[x]_{\varepsilon_{n}} \cdots[x]_{\varepsilon_{n}^{n-1}}, \operatorname{deg} u_{n}=2 n$, где $\varepsilon_{n}$ - первообразный корень $n$-й степени из единицы и $[x]_{\varepsilon_{n}}=g^{-1}\left(\varepsilon_{n} g(x)\right)$ - геометрический элемент, получаемьй возведением в степень $\varepsilon_{n}$ в формальной групе $f\left(x_{1}, x_{2}\right)$ геометрического элемента $x$. Как показано в [13], $u_{n} \in \Lambda_{\{n\}}[[x]]$, где $\Lambda_{\{n\}}=\Lambda \otimes \mathbb{Z}_{\{n\}}$ и $\mathbb{Z}_{\{n\}} \subset \mathbb{Q}$ - кольцо рациональных чисел, знаменатели которых взаимно просты со 
всеми простыми $p$, для которых уравнение $x^{n}=1$ имеет в точности $n$ различных корней в кольце целых $p$-адических чисел. Например, при $n=2$ мы имеем $\mathbb{Z}_{\{2\}}=\mathbb{Z}$ и в этом случае мы получаем $\Lambda$-представление для $u=x \bar{x}, \operatorname{deg} u=4$ (см. вьше), связанное с двузначной формальной группой. Одномерные градуированно корректные $\Lambda_{\{n\}}$-представления алгебры $S$ на $P=\Lambda_{\{n\}}\left[\left[u_{n}\right]\right]$, где $\operatorname{deg} u_{n}=2 n$, связаны с $n$-значньми формальными группами, впервые замеченными также в работе [11]. Первые результаты по алгебраической теории были получены в [14]. Современное состояние теории многозначных групп см. в [15].

Полезный пример одномерного $\mathbb{Z}$-представления алгебры Хопфа $S$, где $\operatorname{dim} u=2$, не эквивалентного действию на геометрическом элементе, извлекается из работы [7]:

$$
s_{(1)}(u)=u^{2}, \quad s_{(n)}(u)=0, \quad n \geqslant 2
$$

(напомним, что на геометрическом элементе представление является другим, см. (4), - таким, что $\left.s_{(n)}(u)=u^{n+1}\right)$. Непосредственно из (11) следует что это представление алгебры $S$ на $P=\mathbb{Z}[u]$ определяется дифференциальными операторами

$$
s_{(1)} \rightarrow u^{2} \frac{d}{d u}, \quad s_{(n)} \rightarrow 0, \quad n \geqslant 2
$$

Образ алгебры Хопфа $S$ над $\mathbb{Z}$ при этом порожден операторами $\frac{1}{n !}\left(u^{2} d / d u\right)^{n}$.

Выделим особо такие представления, где $P$ есть алгебра полиномов от образующей отрицательной размерности, $P=\mathbb{Z}[u](\mathbb{Q}[u])$ или $P=\Lambda[u](\Lambda \otimes \mathbb{Q}[u]), \operatorname{dim} u=$ $-2 q<0$.

ПримеР. Пусть задан базисньй элемент $u \in \Lambda=\Omega^{U}$ размерности $(-2)$ или $(-4)$. Для $u \in \Lambda^{-2}$ мы берем $u=\left[\mathbb{C} P^{1}\right]$. Для $u \in \Lambda^{-4}$ мы берем такое $u$, что $s_{(1)}(u)=0$. Здесь $u=3\left[\mathbb{C} P^{1}\right]^{2}-4\left[\mathbb{C} P^{2}\right]$. Действие алгебры $S$ на элемент $u$ сводится к числам $s_{w}(u) \in \mathbb{Z}, \operatorname{deg} w=2$. Возникает одномерное $\mathbb{Z}$-представление алгебры $S$.

\section{2. Операторы деления и умножения}

Перейдем теперь к построению алгебраического аппарата, позволяющего получать примеры стабильных ассоциативных умножений в комплексных кобордизмах. Все вычисления мы будем проводить в категории модулей над некоторьм коммутативным ассоциативным кольцом $K$ ("числа") с единицей $1 \in K$, причем сами модули будут также коммутативными ассоциативными кольцами $R$ с единицей 1 и $K \subset R$. Мы предполагаем, что $K$ и $R$ не имеют делителей нуля. Все операторы ниже будут $K$-линейны.

ОПРЕДЕЛЕНИЕ 3 . Линейный оператор $\partial: R \rightarrow R$ называется разностной производной, если он не является тождественно нулевым и удовлетворяет тождеству

$$
\partial(x y)=(\partial x) y+x(\partial y)-\alpha(\partial x)(\partial y)
$$

где $\alpha \in R$ - некоторый фиксированньй элемент, необратимый в $R$.

Непосредственно из (12) вытекает, что при наших предположениях $\partial(1)=0$ для любого оператора разностной производной.

Оператор $\pi$ называется мультипликативным, если $\pi(x y)=\pi(x) \pi(y)$. 
Лемма 4. Линейньй оператор д является оператором разностной производной, если и только если оператор $\pi=1-\alpha \partial$ мультипликативен.

ДокАЗАТЕЛЬСТво. Пусть $\partial$ - оператор разностной производной. Тогда по определению мы имеем

$$
\begin{aligned}
\pi(x y) & =x y-\alpha \partial(x y)=x y-\alpha(\partial x) y-\alpha x(\partial y)+\alpha^{2}(\partial x)(\partial y) \\
& =(x-\alpha \partial x)(y-\alpha \partial y)=\pi(x) \pi(y) .
\end{aligned}
$$

Обратное утверждение также проверяется непосредственно. Тем самым, лемма 4 доказана.

Из леммы 4 вытекает мотивировка термина оператор разностной производной.

ЛЕмма 5. Если Ә - оператор разностной производной такой, что $\partial^{2}=\gamma \partial$, əде $\gamma \in \mathbb{R}$, то $(1-\alpha \gamma) \partial(\alpha)=2-\alpha \gamma$. В частности, если $\partial^{2}=0$, то $\partial(\alpha)=2$.

ДОкАЗАТЕЛЬСТво. По определению имеем:

$$
\begin{aligned}
\partial^{2}(x y)= & \partial((\partial x) y+x(\partial y)-\alpha(\partial x)(\partial y)) \\
= & \left(\partial^{2} x\right) y+(\partial x)(\partial y)-\alpha\left(\partial^{2} x\right)(\partial y)+(\partial x)(\partial y)+x\left(\partial^{2} y\right)-\alpha(\partial x)\left(\partial^{2} y\right) \\
& -(\partial \alpha)(\partial x)(\partial y)-\alpha \partial((\partial x)(\partial y))+\alpha(\partial \alpha) \partial((\partial x)(\partial y)) .
\end{aligned}
$$

Из условия $\partial^{2}=\gamma \partial$, получаем

$$
(1-\alpha \gamma)[(1-\alpha \gamma) \partial(\alpha)-(2-\alpha \gamma)] \partial x \partial y=0 .
$$

Так как $\partial$ не есть тождественно нулевой оператор, делителей нуля нет в кольце $R$ и $\alpha$ необратимый элемент, то это тождество дает доказательство леммы.

Лемма 6. Оператор разностной производной д удовлетворяет условию $\partial^{2}=$ $\gamma \partial$, если и только если $\pi^{2}=1 u \pi(\alpha)=-\alpha /(1-\alpha \gamma)$, где $\pi=1-\alpha \partial$.

ДокАЗАТЕЛЬСтво. Согласно лемме 4 оператор $\pi$ - мультипликативен, поэтому:

$$
\begin{aligned}
\pi^{2} x & =\pi(x-\alpha \partial x)=\pi(x)-\pi(\alpha) \pi(\partial x) \\
& =x-\alpha \partial x-\pi(\alpha) \partial x+\alpha \pi(\alpha) \partial^{2} x=x-(\alpha+\pi(\alpha)) \partial x+\alpha \pi(\alpha) \partial^{2} x .
\end{aligned}
$$

Пусть $\partial^{2}=\gamma \partial$. Тогда согласно лемме 5 мы имеем: $\partial(\alpha)=(2-\alpha \gamma) /(1-\alpha \gamma)$ и, следовательно, $\pi(\alpha)=\alpha-\alpha \partial \alpha=-\alpha /(1-\alpha \gamma)$. Таким образом, из $\partial^{2}=\gamma \partial$ вытекает, что $\pi^{2} x=x$ для всех $x$. Наоборот, если $\pi^{2}=1$ и $\pi(\alpha)=-\alpha /(1-\alpha \gamma)$, то согласно (13) получаем, что $\partial^{2}=\gamma \partial$. Лемма доказана.

В рассматриваемых здесь конструкциях содержательно кольцо $R$ моделируется как кольцо функций на каком-то пространстве, мультипликативный оператор $\pi$ моделируется как оператор типа сдвига самого пространства или какого-то другого его гомеоморфизма. Правда, такая интерпретация хороша, если оператор $\pi$ обратим. Этот оператор $\pi$ в важных примерах оказьвается проектором $\pi^{2}=\pi$. Для построения мультипликативных проекторов в [5; с. 887], вводились "операторы деления на элемент $\alpha$ " исходя из тождества (12) и требования $\partial(\alpha)=1$. В [16] была получена классификация всех таких операторов (см. лемму 12 ниже) и показано, что все построеншые позже известные проекторы (Адамса и Квиллена) в теории комплексных кобордизмов получаются как композиции операторов Новикова деления на элемент $\alpha$, для соответствующим образом выбранного множества элементов $\alpha$. 
Лемма 7. Оператор разностной производной д является оператором деления на $\alpha$ (т.е. $\partial(\alpha)=1)$, если и только если композиция операторов да есть единичный оператор и, таким образом,

$$
\pi(x)=(\partial \alpha-\alpha \partial)(x)=[\partial, \alpha](x) .
$$

ДоКАЗАТЕЛЬство. По определению мы имеем

$$
\partial \alpha(x)=\partial(\alpha x)=(\partial(\alpha)) x+\alpha(\partial x)-\alpha(\partial(\alpha))(\partial x) .
$$

Пусть $\partial(\alpha)=1$, тогда $\partial \alpha x=x+\alpha(\partial x)-\alpha(\partial x)=x$. Обратно, пусть мы имеем условие $\partial(\alpha)=1$ или $\partial(\alpha x)=x$. Отсюда получаем

$$
\partial(\alpha x)=(\partial(\alpha)) x+\alpha(\partial x)-\alpha \partial(\alpha)(\partial x)=x .
$$

Следовательно,

$$
(1-\partial(\alpha)) x=\alpha(\partial x)(1-\partial(\alpha))
$$

или

$$
(1-\partial(\alpha))[x-\alpha(\partial x)]=0 .
$$

Полагая в (14) $x=1$ и используя равенство $\partial(1)=0$, получаем, что $\partial(\alpha)=1$. Лемма доказана.

Во всех важных для нас примерах колец $R$ они удовлетворяют следующему условию: если $\alpha \in R$ - необратимьй элемент, то $\bigcap_{n}\left(\alpha^{n} R\right)=0$, где $(\alpha R) \subset R$ - идеал, порожденный элементом $\alpha$. Будем предполагать, что это условие выполняется для рассматриваемых ниже колец $R$.

Лемма 8. Пусть д - оператор разностной производной. Тогда мультипликативный оператор $\pi=1-\alpha \partial$ имеет нетривиальное ядро, если и только если Ә является оператором деления.

ДокАЗАтЕльство. Пусть $\partial$ - оператор деления, т.е. $\partial(\alpha)=1$. Тогда $\pi(\alpha)=$ $\alpha-\alpha \partial(\alpha)=0$. Пусть теперь $\partial(\alpha) \neq 1$ и сушествует элемент $x \neq 0$ такой, что $\pi(x)=0$. Для такого $x$ имеем:

$$
x=\alpha \partial x .
$$

Применяя оператор д, получаем

$$
\partial x=\partial(\alpha) \partial x+\alpha \partial^{2} x-\alpha \partial(\alpha) \partial^{2} x
$$

Следовательно,

$$
(1-\partial(\alpha)) \partial x=\alpha(1-\partial(\alpha)) \partial^{2} x
$$

Так как $\partial(\alpha) \neq 1$, то

$$
\partial x=\alpha \partial^{2} x .
$$

По индукции получаем, что для любого целого $k \geqslant 0$ имеет место соотношение

$$
\partial^{k} x=\alpha \partial^{k+1} x
$$

из которого вытекает, что

$$
x=\alpha^{k} \partial^{k} x
$$

для всех $k \geqslant 0$, т.е. $x \in \bigcap_{k}\left(\alpha^{k} R\right)$. Но в наших кольцах это возможно, только если $x=0$. Лемма доказана. 
СлЕДСТВИЕ 2. Пусть Ә - оператор разностной производной. Мультипликативный оператор $\pi=1-\alpha$ является проектором, $\pi^{2}=\pi$, если и только если $\partial$ - оператор деления на әлемент $\alpha$.

ДокАЗАтЕльство. Пусть $\partial$ - оператор деления на элемент $\alpha$, тогда $\pi(\alpha)=0$ и $\pi^{2} x=\pi(x-\alpha \partial x)=\pi x$. Обратно, пусть $\pi^{2}=\pi$. Тогда $\pi(\pi x-x)=0$ для любого $x$, и согласно лемме 8 мы получаем, что $\pi x=x$. Но это невозможно, так как $\alpha \neq 0$. Следствие доказано.

ЛЕмма 9. При фиксированном $\alpha \in R$ множсество мультипликативных операторов $\Pi_{\alpha}(R)$, состоящее из таких $\pi \in \Pi_{\alpha}(R)$, что $\pi=1-\alpha \partial$, образует подполугруппу в полугруппе $\Pi(R)$ всех мультипликативных операторов $R \rightarrow R$ относительно операчии суперпозичии $\left(\pi_{1} \circ \pi_{2}\right)(u)=\pi_{1}\left(\pi_{2}(u)\right)$. Для соответствующих операторов разностной производной эта операция имеет вид

$$
\partial_{1} \circ \partial_{2}=\partial_{1}+\partial_{2}-\partial_{1}\left(\alpha \partial_{2}\right), \quad 1-\alpha \partial_{i}=\pi_{i}, \quad i=1,2 .
$$

ДокАЗАТЕльСТво. По определению имеем:

$$
\left(\pi_{1} \circ \pi_{2}\right)(u)=u-\alpha \partial_{1} \circ \partial_{2}(u) .
$$

Тогда

$$
\begin{aligned}
\alpha \partial_{1} \circ \partial_{2}(u) & =u-\pi_{1} \circ \pi_{2}(u)=u-\pi_{1}\left(\pi_{2}(u)\right)=u-\pi_{1}\left(u-\alpha \partial_{2}(u)\right) \\
& =u-\left(u-\alpha \partial_{2}(u)\right)+\alpha \partial_{1}\left[u-\alpha \partial_{2}(u)\right]=\alpha\left[\partial_{1}(u)+\partial_{2}(u)-\partial_{1}\left(\alpha \partial_{2}\right)(u)\right] .
\end{aligned}
$$

Следовательно,

$$
\partial_{1} \circ \partial_{2}=\partial_{1}+\partial_{2}-\partial_{1}\left(\alpha \partial_{2}\right)
$$

Лемма доказана.

Приведем некоторые известные примеры операторов разностной производной.

1) Пусть $R$ - кольцо рядов от переменной $x, R=K[[x]]$. Фиксируем $\alpha(x)$ и $\psi(x)$ из $R$, где $\alpha(0)=0$.

Положим для $p \in R$

$$
\partial p(x)=\frac{p(x)-p(x-\alpha(x) \psi(x))}{\alpha(x)} .
$$

Этот оператор всегда обладает свойством (12), т.е. это - оператор разностной производной. Очевидно, мы получаем

$$
\pi p(x)=p(x)-\alpha \frac{p(x)-p(x-\alpha(x) \psi(x))}{\alpha}=p(x-\alpha(x) \psi(x)) .
$$

Это всегда обычный сдвиг аргумента.

2) Пусть $R=K[[x, y]]$ и $\alpha=x-y$. Мы полагаем

$$
\partial p(x, y)=\frac{p(x, y)-p(y, x)}{x-y}
$$

Здесь $\pi(p(x, y))=p(y, x)$, т.е. $\pi^{2}=1$ и $\partial^{2}=0$. 
Для $R=K\left[\left[x_{1}, \ldots, x_{n}\right]\right]$ определяются операторы $\partial_{i j}$, где

$$
\partial_{i j} p\left(x_{1}, \ldots, x_{n}\right)=\frac{p\left(\ldots x_{i} \ldots x_{j} \ldots\right)-p\left(\ldots x_{j} \ldots x_{i} \ldots\right)}{x_{i}-x_{j}} .
$$

Эти операторы нашли важное приложение в теории когомологий многообразий флагов [17]. Они часто используются и назьваются "divided difference operators". Их алгебра очень красива.

Пусть $f(x, y) \in K[[x, y]]$ - ряд, задаюший некоторую формальную группу над $K$. Возьмем $\alpha=f(x, \bar{y})$, где $\bar{y}$ - обратный элемент в формальной группе $f(x, y)$. Формула

$$
\partial p(x, y)=\frac{p(x, y)-p(y, x)}{f(x, \bar{y})}
$$

задает оператор разностной производной в $R=K[[x, y]]$. Здесь $\pi(p(x, y))=p(y, x)$, $\pi^{2}=1$ и $\partial^{2}=\gamma \partial$, где $\gamma \in K[[x, y]]$. Так, при $f(x, y)=x+y-a x y, a \in K$, мы имеем $\partial^{2}=a \partial$. Операторы (17), где $f(x, y)$ - формальная группа геометрических элементов, нашли приложения в теории комплексных кобордизмов [18], [19].

Примеры 1) и 2) дают частные случаи следуюшей конструкции.

Пусть $R=K\left[\left[x_{1}, \ldots, x_{n}\right]\right]$. Фиксируем $\alpha(x) \in R$, где $\alpha(0)=0$, и вектор-функцию

$$
\psi(x)=\left(\psi_{1}(x), \ldots, \psi_{n}(x)\right),
$$

где $\psi_{k}(x) \in R, k=1, \ldots, n$. Здесь $x=\left(x_{1}, \ldots, x_{n}\right)$.

Положим для $p \in R$ :

$$
\partial p(x)=\frac{1}{\alpha(x)}(p(x)-p(x-\alpha(x) \psi(x))) .
$$

Получаем:

$$
\pi p(x)=p(x-\alpha(x) \psi(x)) .
$$

Наибольшие приложения нашли случаи, когда $\alpha(x)=\langle x, \xi\rangle=\sum_{i=1}^{n} x_{i} \xi^{i}$ и

(i) $\psi(x)=\frac{\xi}{\langle\xi, \xi\rangle}$, здесь $\pi_{\xi} p(x)=p\left(x-\langle x, \xi\rangle \frac{\xi}{\langle\xi, \xi\rangle}\right)-$ проектор, $\pi_{\xi}^{2}=\pi_{\xi}$ и $\partial=\partial_{\xi}-$ оператор деления на $\langle x, \xi\rangle$,

(ii) $\psi(x)=2 \frac{\xi}{\langle\xi, \xi\rangle}$, здесь $\pi_{\xi} p(x)=p\left(x-2\langle x, \xi\rangle \frac{\xi}{\langle\xi, \xi\rangle}\right)$ удовлетворяет условию $\pi_{\xi}^{2}=1$ и $\partial_{\xi}^{2}=0$.

Это операторы, определяемые отражениями относительно подпространств $V=$ $\{x \mid\langle x, \xi\rangle=0\}$. Они используются при определении известных операторов Данкла [20].

Выбирая соответствуюшие конфигурации векторов $\xi_{1}, \ldots, \xi_{k}$, получают интересные алгебры операторов $\partial_{\xi_{l}}, l=1, \ldots, k$.

3) Пусть $\phi: R \rightarrow R$ - кольцевой гомоморфизм. Согласно [21] $\phi$-дифференцированием алгебры $R$ называется такой оператор $\delta: R \rightarrow R$, что

$$
\delta(a b)=\phi(a) \delta(b)+\delta(a) b .
$$


ОПРеДЕЛЕниЕ 4. Расширением Оре кольца $R$ назьвается алгебра, аддитивно изоморфная $R[t]$, такая, что $R \subset R[t]$ и

$$
t a=\phi(a) t+\delta(a)
$$

Имеет место

Лемма 10. Оператор разностной производной $\partial$, удовлетворяющий тождеству (12):

$$
\partial(a b)=(\partial a) b+a(\partial b)-\alpha(\partial a)(\partial b),
$$

$\alpha \in R$, является ф-дифференцированием Оре, где $\phi=\pi=1-\alpha \partial: R \rightarrow R$.

Доказательство леммы получается прямой элементарной проверкой.

Перейдем теперь к построению новых $K$-билинейных ассоциативных умножений в кольце $R$.

Teopema 1. Onерация

$$
\mu_{1}(x, y)=\pi_{1}(x) \pi_{2}(y)=x y-\alpha_{1} \partial_{1}(x) y-\alpha_{2} \partial_{2}(y) x+\alpha_{1} \alpha_{2} \partial_{1}(x) \partial_{2}(y),
$$

әде $\partial_{1}, \partial_{2}$ - операторы разностной производной, соответствующие әлементам $\alpha_{1}, \alpha_{2} \in R$, задает ассоциативное умножение, если и только если операторы $\partial_{1}$ и $\partial_{2}$ являются операторами деления, и соответствующие мультипликативныее операторы $\pi_{1}$ и $\pi_{2}$ коммутируют, т.е. $\pi_{1} \pi_{2}=\pi_{2} \pi_{1}$. В частности, каждий оператор деления д задает ассоциативное умножение $\mu(x, y)=\pi(x) \pi(y)$, где $\pi=1-\alpha \partial$. Умножение $\mu_{1}(x, y)$ коммутативно, если и только если $\pi_{1}=\pi_{2}$.

ДОКАЗАТЕЛЬСТВО. Положим

$$
x \circ y=\mu_{1}(x, y)=\pi_{1}(x) \pi_{2}(y) .
$$

Тогда

$$
(x \circ y) \circ z=\pi_{1}(x \circ y) \pi_{2}(z)=\pi_{1}^{2}(x) \pi_{1}\left(\pi_{2}(y)\right) \pi_{2}(z) .
$$

Пусть операторы разностной производной $\partial_{1}$ и $\partial_{2}$ являются операторами деления, т.е. $\partial_{1}\left(\alpha_{1}\right)=\partial_{2}\left(\alpha_{2}\right)=1$ и, следовательно, $\pi_{1}^{2}=\pi_{1}, \pi_{2}^{2}=\pi_{2}$. В этом случае при дополнительном условии $\pi_{1} \pi_{2}=\pi_{2} \pi_{1}$ мы получаем, используя (21), что умножение $\mu_{1}$ ассоциативно.

Наоборот, пусть $\mu_{1}$ - ассоциативное умножение, тогда из условия

$$
(x \circ 1) \circ 1=x \circ(1 \circ 1)
$$

получаем:

$$
\pi_{1}^{2}(x) \pi_{1}\left(\pi_{2}(1)\right) \pi_{2}(1)=\pi_{1}(x) \pi_{2}\left(\pi_{1}(1)\right) \pi_{2}^{2}(1) .
$$

Следовательно, $\pi_{1}^{2}=\pi_{1}$, так как $\pi(1)=1$ для любого мультипликативного оператора $\pi$. Аналогично получаем, что $\pi_{2}^{2}=\pi_{2}$.

Из условия: $(1 \circ y) \circ 1=1 \circ(y \circ 1)$ вытекает, что $\pi_{1} \pi_{2}=\pi_{2} \pi_{1}$.

Используя теперь следствие 2 , получаем, что операторы $\partial_{1}$ и $\partial_{2}$, соответствуюшие операторам $\pi_{1}$ и $\pi_{2}$, являются операторами деления.

И наконец, если умножение $\mu_{1}(x, y)$ коммутативно, то из условия $x \circ 1=1 \circ x$ получаем, что это возможно, если и только если $\pi_{1}=\pi_{2}$. Теорема доказана. 
Teopema 2. Onерация

$$
\mu_{2}(x, y)=x y+\beta \partial(x) \partial(y), \quad \beta \in R,
$$

где $\partial$ - оператор разностной производной, соответствующий әлементу $\alpha \in R$ и $\beta$ некоторый әлемент из $R$, задает ассоииативное умножение, если и только если выполнено одно из следующих условий:

(i) оператор д является оператором деления и $\pi(\beta)=0$, где $\pi=1-\alpha \partial$;

(ii) оператор д не является оператором деления и $\partial^{2} x \partial y=\partial x \partial^{2} y$ для любых әлементов $x, y \in R$.

В частности, условие (ii) выполняется, если $\partial^{2}=\gamma \partial$.

Обратим внимание, что условие іi) не зависит от выбора $\beta$, т.е. $\beta$ в этом случае представляет собой независимый параметр деформации, связывающий исходное умножение в кольие $R$ с новым умножением.

ДокАЗАТЕЛЬСТво. Положим

$$
x \circ y=\mu_{1}(x, y)=x y+\beta \partial x \partial y .
$$

Тогда

$$
\begin{aligned}
(x \circ y) \circ z= & (x y+\beta \partial x \partial y) z+\beta \partial(x y+\beta \partial x \partial y) \partial z \\
= & x y z+\beta((\partial x \partial y) z+(\partial x) y \partial z+x \partial y \partial z) \\
& -\beta \alpha \partial x \partial y \partial z+\beta(\partial \beta \partial x \partial y \partial z+\beta \partial(\partial x \partial y) \partial z-\alpha \partial \beta \partial(\partial x \partial y) \partial z) .
\end{aligned}
$$

Следовательно, умножение $\mu_{2}$ ассоциативно, если и только если выполняется соотношение

$$
(\beta-\alpha \partial \beta)(\partial(\partial x \partial y) \partial z-\partial x \partial(\partial y \partial z))=0
$$

для всех $x, y, z \in R$.

Пусть $\partial$ является оператором деления, т.е. $\partial(\alpha)=1$. Возьмем $x=\alpha^{2}, y=z=\alpha$. Имеем: $\partial\left(\alpha^{2}\right)=2 \alpha \partial \alpha-\alpha(\partial \alpha)^{2}=\alpha$ и, следовательно, $\partial^{2}\left(\alpha^{2}\right)=1$. При выбранных значениях $x, y, z$, учитьвая, что $\partial(1)=0$, соотношение (23) переходит в соотношение $\pi(\beta)=\beta-\alpha \partial \beta=0$. Утверждение (i) доказано.

Пусть теперь $\partial$ не является оператором деления. Тогда согласно лемме 8 имеем: $\pi(\beta) \neq 0$, если $\beta \neq 0$. Так как

$$
\partial(\partial x \partial y)=\partial^{2} x \partial y+\partial x \partial^{2} y-\alpha \partial^{2} x \partial y,
$$

то простая выкладка показьвает, что условие (23) эквивалентно условию:

$$
\left(\partial^{2} x \partial z-\partial z \partial^{2} z\right)\left(\partial y-\alpha \partial^{2} y\right)=0
$$

для всех $x, y, z \in R$. Заметим, что $\partial y-\alpha \partial^{2} y=\pi \partial y$. Так как, по предположению, $\pi$ не является оператором деления, то условие $\pi \partial(y)=0$ эквивалентно условию $\partial y=0$ для всех $y \in R$, что невозможно. Следовательно, условие (24) эквивалентно условию:

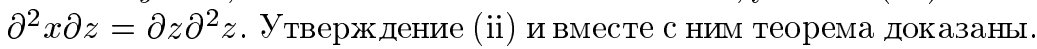

Приведем еще одну чисто алгебраическую конструкцию нового ассоциативного умножения, подсказанную теорией комплексных кобордизмов (см. [22]). 
Теорема 3. Пусть П: $R \rightarrow R u \delta: R \rightarrow R$ - линейнъе операторы такие, что

1) $\Pi^{2}=\Pi, \delta \Pi=\delta$,

2) $\delta(\Pi x \Pi y)=\delta x(\Pi y)+(\Pi x) \delta y-\alpha(\delta x)(\delta y)$,

3) $\Pi((\Pi x)(\Pi y))=(\Pi x)(\Pi y)+\beta(\delta x)(\delta y)$

для некоторых әлементов $\alpha$ и $\beta$ из $R$. Тогда операция

$$
\mu(x, y)=\Pi((\Pi x)(\Pi y))=(\Pi x)(\Pi y)+\beta(\delta x)(\delta y)
$$

задает ассоциативное умножение в кольце $R$.

ДокАЗАТЕЛЬСТво. Положим $\mu(x, y)=x * y$. Тогда по определению имеем:

$$
\begin{aligned}
(x * y) * z & =\Pi(x * y) \Pi z+\beta \delta(x * y) \delta z=(\Pi(\Pi x \Pi y)) \Pi z+\beta \delta(\Pi((\Pi x)(\Pi y))) \delta z \\
& =\Pi x \Pi y \Pi z+\beta(\delta x)(\delta y) \Pi z+\beta[\delta x(\Pi y) \delta z+(\Pi x) \delta y \delta z-\alpha(\delta x)(\delta y)(\delta z)] \\
& =\Pi x \Pi y \Pi z+\beta[\delta x \delta y \Pi z+\delta x \Pi y \delta z+\Pi x \delta y \delta z]-\beta \alpha \delta x \delta y \delta z=x *(y * z) .
\end{aligned}
$$

Теорема доказана.

\section{3. Примеры из теории кобордизмов}

В этом разделе мы построим новые умножения в теории комплексных кобордизмов, опираясь на полученные выше результаты.

Напомним, что мультипликативные операторы в алгебре $A^{U}=(\Lambda S)^{\wedge}$ полностью определяются своим действием на геометрических элементах $x \in U^{2}(X)$, т.е. все они задаются произвольными рядами вида

$$
\phi(x)=x+\sum_{i \geqslant 1} \phi_{i} x^{i+1}, \quad \phi_{i} \in \Lambda .
$$

Мультипликативньй оператор $\phi$, задаваемый рядом $\phi(x)$, действует на произвольном элементе $y \in U^{*}(X)$ по формуле:

$$
\phi(y)=y+\sum_{\operatorname{deg} w>0} \phi_{w} s_{w}(y)
$$

где $\phi_{w}=\phi_{1}^{k_{1}} \cdots \phi_{l}^{k_{l}}$ для $w=\left(k_{1}, \ldots, k_{l}\right)$.

ЛЕмма 11. Ряд $\phi(x)=x+\sum_{i \geqslant 1} \phi_{i} x^{i+1}$ задает мультипликативньй проектор $\phi \in A^{U}$, m.е. $\phi^{2}=\phi$ в $A^{U}$, если и только если $\phi\left(\phi_{i}\right)=\phi_{i}+\sum \phi_{w} s_{w}\left(\phi_{i}\right)=0 \partial л я$ всех $i \geqslant 1$.

ДокАЗАТЕльство. Имеем на геометрическом элементе $x \in U^{2}(X)$

$$
\phi(\phi(x))=\phi(x)+\sum \phi\left(\phi_{i}\right) \phi(x)^{i+1} .
$$

Следовательно, $\phi(\phi(x))=\phi(x)$, если и только если $\sum_{i \geqslant 1} \phi\left(\phi_{i}\right) t^{i+1}=0$ в кольце $\Lambda[[t]]$, где $t=\phi(x)$. Это эквивалентно условию: $\phi\left(\phi_{i}\right)=0, i \geqslant 1$. Лемма доказана.

Мультипликативньй оператор $\phi \in A^{U}$ с $\phi(x)=x+\sum_{i \geqslant 1} \phi_{i} x^{i+1}, \phi_{i} \in \Lambda$, на геометрическом элементе $x \in U^{2}(X)$ называется однородным. если $\operatorname{deg} \phi_{i}=-2 i$. Множество всех однородных мультипликативных операторов образует полугрупп, в которой лежат проекторы, важнейшие для теории кобордизмов.

Для целого числа $m$ положим $\mathbb{Z}_{(m)}=\mathbb{Z}\left[m^{-1}\right]$. Из [16] извлекается следующий результат. 
Лемма 12. Пусть $\alpha \in \Lambda^{-2 n} u s_{(n)} \alpha=m \neq 0, m \in \mathbb{Z}$. Onepamop $\partial \in A^{U}$ задает оператор деления на $\alpha$ в кольче комплексньх кобордизмов $U^{*}(X) \otimes \mathbb{Z}_{(m)}$ такой, что мультипликативный оператор $\pi, \pi=1-\alpha \partial$, является однородным, если и только если его значение на геометрическом кобордизме имеет вид:

$$
\partial x=\frac{1}{m} x^{n+1}+\sum_{i \geqslant 1} a_{i} x^{n+i+1}, \quad a_{i} \in \Lambda^{-2 i} \otimes \mathbb{Z}_{(m)},
$$

где $\left(a_{i}\right)$ - свободный набор параметров оператора д.

ДокАЗАТЕльство. Согласно предыдущим алгебраическим результатам достаточно проверить только, что $\pi(\alpha)=0$. Имеем: $\pi(x)=x-\alpha x^{n}\left(\frac{1}{m} x+\sum_{i \geqslant 1} a_{i} x^{i+1}\right)$, где $x$ - геометрический элемент. Таким образом, $\pi(y)=y-\alpha \frac{1}{m} s_{(n)} y+$ (члены, содержащие $\left.s_{w}(y), \operatorname{deg} w>2 n\right)$. Следовательно, $\pi(\alpha)=\alpha-\alpha \frac{1}{m} s_{(n)}(\alpha)=0$. Лемма доказана.

Используя лемму 12, можно описать все пары операторов деления $\partial_{1}$ и $\partial_{2}$ такие, что соответствующие мультипликативные проекторы $\pi_{1}$ и $\pi_{2}$ являются однородными и коммутирующими. Таким образом, согласно теореме 1 мы получаем множество ассоциативных умножений вида $\mu_{1}(x, y)=\pi_{1}(x) \pi_{2}(y)$ в комплексных кобордизмах.

Далее, для каждого оператора деления $\partial$ из леммы 12 мы можем взять любой элемент $\beta$ из ядра оператора $\pi=1-\alpha \partial$ и получить согласно п. (i) теоремы 2 ассоциативное умножение вида $\mu_{2}(x, y)=x y+\beta \partial x \partial y$.

Следуюший результат позволяет строить ассоциативные умножения вида $\mu_{2}(x, y)$ на основании п. (ii) теоремы 2.

Лемма 13. Пусть $\alpha \in \Lambda^{-2 n} u s_{(n)}(\alpha)=2 n$. Мультипликативная однородная операция $\pi \in A^{U} \otimes \mathbb{Z}_{(n)}$, которая на геометрическом әлементе $x \in U^{2}(X)$ действует по формуле

$$
\pi(x)=x-\alpha \partial x=\frac{x}{\sqrt[n]{1+\alpha x^{n}}}
$$

задает оператор разностной производной д такой, что $\partial^{2}=0$.

ДокАЗАТЕЛЬСтво. Согласно лемме 6 достаточно показать, что

$$
\pi(\alpha)=-\alpha \text { и } \pi^{2}=1 .
$$

Имеем:

$$
\pi(x)=x-\frac{1}{n} \alpha x^{n+1}+O\left(x^{2 n+1}\right) .
$$

Таким образом, $\pi(y)=y-\frac{1}{n} \alpha s_{(n)}(y)+\left(\right.$ члены, содержашие $\left.s_{w}(y), \operatorname{deg} w>2 n\right)$. Следовательно, $\pi(\alpha)=\alpha-\frac{1}{n} \alpha \cdot 2 n=-\alpha$. Так как $\pi^{2}$-мультипликативньй оператор, то он определяется своим значением на геометрическом элементе $x$. Имеем:

$$
\pi^{2}(x)=\pi\left(\frac{x}{\sqrt[n]{1+\alpha x^{n}}}\right)=\frac{\pi(x)}{\sqrt[n]{1+\pi(\alpha) \pi(x)^{n}}}=\frac{x}{\sqrt[n]{1+\alpha x^{n}}} \cdot \frac{1}{\sqrt[n]{1-\alpha \frac{x^{n}}{1+\alpha x^{n}}}}=x
$$

Итак, $\pi^{2}(x)=x$ и, следовательно, $\pi^{2}=1$. Лемма доказана. 
ПримеР. Пусть [ $\left.\mathbb{C} P^{m}\right]-$ класс комплексных кобордизмов комплексного $m$-мерного проективного пространства. Имеем, $s_{(m)}\left[\mathbb{C} P^{m}\right]=-(m+1)$. Таким образом, взяв в качестве $\alpha$ класс кобордизмов - $\left[\mathbb{C} P^{2 n-1}\right]$, мы получаем согласно лемме 13 оператор разностной производной $\partial$ на кольцах $U^{*}(X) \otimes \mathbb{Z}_{(n)}$ такой, что $\partial^{2}=0$, и, следовательно, новое ассоциативное умножение с свободньм параметром $\beta$. В случае комплексной проективной прямой $\mathbb{C} P^{1}$ мы получаем такое умножение на кольцах $U^{*}(X)$.

В заключение опишем пример ассоциативного умножения в кольцах $U^{*}(X)$, основанньй на конструкции из теоремы 3 .

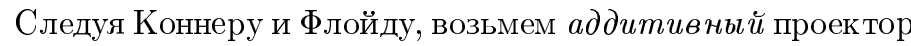

$$
\Pi: U^{*}(X) \rightarrow U^{*}(X)
$$

который полностью задается следуюшим действием на классах кобордизмов замкнутых $U$-многообразий:

пусть $\xi \rightarrow \mathbb{C} P^{1}$ - каноническое комплексное одномерное расслоение над проективной прямой $\mathbb{C} P^{1}$. Рассмотрим замкнутое $U$-многообразие $M^{2 n}$ со стабильно комплексным касательным расслоением $\tau$. По определению, П $\left(\left[M^{2 n}\right]\right)$ - это класс комплексных кобордизмов подмногообразия $i: \widehat{M}^{2 n} \subset M^{2 n} \times \mathbb{C} P^{1}$ с нормальным расслоением вложения $i^{*}((\operatorname{det} \tau) \otimes \xi)$, где $\operatorname{det} \tau$ - детерминантное расслоение комплексного расслоения $\tau$.

Действие проектора $\Pi \in A^{U}$ на классе Тома $x_{n}$ комплексного $n$-мерного расслоения $\eta \rightarrow X$ вычисляется по формуле:

$$
\Pi\left(x_{n}\right)=x_{n}+\sum_{i \geqslant 2} \alpha_{i 1} \partial_{i} x_{n},
$$

где $\alpha_{i 1} \in \Lambda^{-2 i}$ - коэффициент при $x^{i} y$ в формальной группе $f(x, y)$ геометрических элементов $x, y$ и $\partial_{i} \in A^{U}$ - операторы, которые на классе Тома $x_{n}$ действуют по формуле:

$$
\partial_{i} x_{n}=x_{n} c_{1}(\overline{\operatorname{det} \eta}),
$$

где $c_{1}$ - первый класс Чженя в комплексных кобордизмах, и ${ }^{-}$- знак комплексного сопряжения.

Взяв в качестве $\delta$ оператор $\partial_{1} \in A^{U}$, можно проверить, что пара П, $\delta$ удовлетворяет всем соотношениям из теоремы 3 (детали вычислений см. в [22], [23]). Следовательно, согласно этой теореме в $U^{*}(X)$ определено ассоциативное умножение, задаваемое формулой:

$$
x * y=\Pi(\Pi x \Pi y) .
$$

Это умножение играет ключевую роль в описании мультипликативной структуры кольца кобордизмов $S U$-многообразий, или, как сейчас стали говорить, многообразий Калаби-Яо. 


\section{СПИСОК ЛИТЕРАТУРЫ}

[1] Novikov S. P. (Ed.). Topology I. Berlin: Springer-Verlag, 1996. (Encyclopaedia of Math. Sci. V. 12.)

[2] Milnor J. W. On the cobordism ring $\Omega^{*}$ and a complex analogue. Part I // Amer. J. Math. 1960. V. 82. P. 505-521.

[3] Новиков С. П. О некоторых задачах топологии многообразий, связанных с теорией пространств Тома // Докл. АН СССР. 1960. Т. 132. № 5. С. 1031-1034.

[4] Новиков С. П. Гомотопические свойства комплексов Тома // Матем. сб. 1962. Т. 57 (99). C. $406-442$.

[5] Новиков С. П. Методы алгебраической топологии с точки зрения теории кобордизмов // Изв. АН СССР. Сер. матем. 1967. Т. 31. С. 855-951.

[6] Landweber P.S. Cobordism operations and Hopf algebras // Trans. Amer. Math. Soc. 1967. V. 129. № 1. P. 94-110.

[7] Бухштабер В.М., Шокуров А.В. Алгебра Ландвебера-Новикова и формальные векторные поля на прямой // Функц. анализ и его прил. 1978. Т. 12. № 3. С. 1-11.

[8] Новиков С.П. Различные удвоения алгебр Хопфа. Алгебры операторов на квантовых группах, комплексные кобордизмы // УМН. 1992. Т. 47. № 5. С. 189-190.

[9] Buchstaber V. M. Semigroups of maps into groups, operator doubles, and complex cobordisms // Amer. Math. Soc. Transl. Ser. 2. 1995. V. 170. P. 9-31.

[10] Бухштабер В. M. Характер Чженя-Дольда в кобордизмах // Матем. сб. 1970. T. 83 (125). C. $576-595$.

[11] Бухштабер В. М., Новиков С. П. Формальные группы, степенные системы и операторы Адамса // Матем. сб. 1971. Т. 84 (126). С. 116-153.

[12] Бухштабер В. М. Характеристические классы в кобордизмах и топологические приложения теорий однозначных и двузначных формальых групп // Итоги науки и техники. Совр. пробл. матем. Т. 10. М.: ВИНИТИ, 1978. С. 5-178.

[13] Бухштабер В. М., Холодов А.Н. Топологические конструкции, связанные с многозначными формальными группами // Изв. АН СССР. Сер. матем. 1982. Т. 46. № 1. С. 3-26.

[14] Холодов А.Н. Алгебраическая теория многозначных формальных групп // Матем. сб. 1981. T. 114. № 2. C. 299-321.

[15] Buchstaber V. M., Rees E. G. Multivalued groups, their representations and Hopf algebras // Transform. Groups. 1997. V. 2. № 4. P. 325-349.

[16] Бухштабер В. М. Когомологические операции и формальная группа в кобордизмах. Характеристические классы в кобордизмах // В. Снэйт. Алгебраический кобордизм и $K$-теория. Приложение 4. М.: Мир, 1983. С. 227-248.

[17] Берштейн И. Н., Гельфанд И. М., Гельфанд С. И. Клетки Шуберта и когомологии пространства $G / P$ // УМН. 1973. Т. 28. №3. С. 3-26.

[18] Bressler P., Evens S. The Schubert calculus, braid relation, and generalized cohomology // Trans. Amer. Math. Soc. 1990. V. 317. № 2. P. 799-811.

[19] Фельдман К. Э. Эквивариантньй аналог теоремы Пуанкаре-Хопфа // Зап. науч. семин. ПОМИ. 2000. Т. 267. С. 160-174.

[20] Dunkl C.F. Differential-difference operators associated to reflection groups // Trans. Amer. Math. Soc. 1989. V. 311. № 1. P. 167-183.

[21] Ore O. Theory of non-commutative polynomials // Ann. of Math. (2). 1933. V. 34. P. $480-508$.

[22] Бухштабер В. М. Проекторы в унитарных кобордизмах, связанные с $S U$-теорией // УМН. 1972. Т. 27. №6. С. 231-232.

[23] Стонг Р. Заметки по теории кобордизмов. М.: Мир, 1973.

Московский государственный университет им. М. В. Ломоносова;

Поступила в редакцию University of Maryland at College Park;

01.06 .2000

Department of Mathematics the University of Oregon

E-mail: buchstab@ftri.extech.msk.su; novikov@ipst.umd.edu, novikov@landau.ac.ru; botvinn@darkwing.uoregon.edu; yuz@darkwing.uoregon.edu 